\section{Prevalência de abuso físico na infância e exposição à violência parental em uma amostra brasileira}

\author{
Prevalence of physical abuse in childhood and \\ exposure to parental violence in a \\ Brazilian sample
}

\author{
1 Universidade Estadual do \\ Centro Oeste, Guarapuava, \\ Brasil. \\ 2 Núcleo de Psiquiatria, \\ Universidade Federal \\ de Santa Catarina \\ Florianópolis, Brasil. \\ 3 Instituto de Psiquiatria \\ de Santa Catarina \\ Florianópolis, Brasil. \\ 4 Departamento de \\ Psiquiatria, Universidade \\ Federal de São Paulo, \\ São Paulo, Brasil. \\ 5 UNIAD - Unidade de \\ Pesquisa em Álcool e Drogas \\ São Paulo, Brasil. \\ 6 University of Texas School \\ of Public Health, \\ Dallas, U.S.A. \\ Correspondência \\ D. V. Zanoti-Jeronymo \\ Universidade Estadual do \\ Centro-Oeste. \\ Rua Vicente Machado 1530, \\ apto. 402, Guarapuava, SP \\ 85010-260, Brasil. \\ danielazanoti@uol.com.br
}

\begin{abstract}
The aim of this study was to investigate the prevalence of physical abuse and exposure to parental violence during childhood, in relation to socio-demographic characteristics. Multi-stage sampling was used to select 3,007 individuals 14 years or older in 2005-2006, from all regions of the country. The study detected cases of physical abuse (beating, striking with objects, burning or scalding, threatening and/or using knives or firearms) and exposure to parental violence (witnessing parental threats and/or actual physical aggression) during childhood. Analyses were performed with the Pearson chi-square test. Prevalence of history of physical abuse in childhood was $44.1 \%$ (33.8\% moderate, 10.3\% severe). Prevalence of exposure to parental violence was $26.1 \%$ (7.5\% witnessed moderate parental violence and $18.6 \%$ severe). Combined analyses of these two types of violence showed significant associations between them. The findings may help implement intervention strategies to call the attention of health professionals to the high prevalence of this type of violence.
\end{abstract}

Domestic Violence; Child Abuse; Parents

\author{
Daniela Viganó Zanoti-Jeronymo ${ }^{1}$ \\ Marcos Zaleski 2,3 \\ Ilana Pinsky 4,5 \\ Raul Caetano 6 \\ Neliana Buzi Figlie 4,5 \\ Ronaldo Laranjeira 4,5
}

\section{Introdução}

A violência doméstica é reconhecida mundialmente como um dos principais problemas sociais e de saúde pública devido a sua elevada ocorrência 1,2 . Suas conseqüências podem ser vistas em diferentes fases da vida. Estudos indicam que crianças que são expostas à violência doméstica são mais agressivas com seus pares ${ }^{3}$, apresentam maior risco para problemas de saúde $4,5 \mathrm{e}$ mais problemas comportamentais e emocionais ainda na infância 6,7,8,9,10,11,12. Outros estudos associam as experiências de violência na infância à violência na adolescência 13,14. Pesquisas indicam que o histórico de violência na infância está associado a níveis mais elevados de distúrbios psicológicos, tais como depressão, ansiedade e abuso de substâncias na idade adulta 15,16,17.

O efeito a longo-prazo, mais relatado na literatura, da exposição à violência parental e abuso físico parental na infância é o risco elevado para perpetração da violência na idade adulta, seja em seus relacionamentos íntimos 16,17,18,19,20,21,22,23, seja com a perpetração contra os próprios filhos $7,13,18,19,24$.

No entanto, comparada aos estudos realizados nos países desenvolvidos, a produção científica nacional sobre violência doméstica ainda é limitada. Estudos de revisão da literatura apontam para uma gradativa intensificação das publicações sobre esse tema nas últimas décadas 25,26. Estes estudos revelam que o foco de interesse das 
produções científicas incluem: análises teóricas da violência; programas de intervenção e prevenção da violência; descrições dos aspectos informativos sobre o impacto da violência; e identificação e descrição dos casos de violência contra crianças, adolescentes e mulheres. No entanto, estes últimos utilizam-se, na sua maioria, de informações disponibilizadas por serviço de atendimento e/ou encaminhamento de vítimas no âmbito dos serviços de saúde, ou seja, apenas indivíduos que procuram por ajuda, o que dificulta a caracterização do fenômeno da violência em nível populacional. Os estudos ainda apontam que estas pesquisas, na maioria regionais, trazem valiosas contribuições para ajudar a dimensionar o problema da violência doméstica.

No que tange aos estudos de prevalência da violência doméstica contra crianças e adolescentes, Bordin et al. 7 realizaram o primeiro estudo de base populacional no Brasil. Com uma amostra probabilística de mães de uma comunidade de baixa renda, investigaram as possíveis relações entre punição física grave e problemas de saúde mental em crianças e adolescentes. As autoras encontraram que a punição física grave é uma prática comum nesta população e que crianças abusadas exibem mais problemas de comportamento quando comparadas a crianças não-abusadas. Vale a pena acrescentar que segundo Straus 27, a punição corporal é o principal fator de risco para o abuso físico. Bordin et al. 7 apontam ainda que os estudos nacionais sobre esta temática, que mais se aproximam de estudos populacionais sobre abuso físico de crianças e adolescentes, tomam como base populações de escolares.

Com relação à prevalência de violência doméstica em outras faixas etárias, recentemente dois estudos foram divulgados. O estudo multicêntrico da Organização Mundial da Saúde sobre saúde da mulher e violência doméstica 28 , de base populacional, analisou a prevalência da violência contra mulheres por parceiros íntimos e a repercussão da violência na saúde de 1.172 mulheres da cidade de São Paulo e 1.473 da Zona da Mata em Pernambuco. Os autores relatam uma prevalência de violência física por parceiros íntimos encontrada no Brasil - 40\% em São Paulo e 37\% na Zona da Mata - não estão entre as mais altas, quando comparada com dados internacionais. No segundo estudo Zalescki et al. 29 analisaram a prevalência da violência íntima do parceiro entre homens e mulheres brasileiros ( $\mathrm{n}=1.445$ casais) e encontraram uma prevalência de $10,7 \%$ de violência íntima homem-a-mulher e uma prevalência de $14,6 \%$ de violência mulher-a-homem.

Estudos apontam que a identificação da violência doméstica é um dos maiores desafios para os pesquisadores da área da saúde, sendo que estimar a sua freqüência e ocorrência é extremamente difícil 4,30,31. No entanto, apesar das dificuldades, a mensuração deste fenômeno tão complexo é necessária para obtenção de estimativas nacionais.

No Brasil, a necessidade de pesquisas de delineamento do fenômeno da violência doméstica ainda mostra-se imprescindível. O desenvolvimento de estudos com base populacional avaliando a prevalência da violência na população geral faz-se necessário para a implementação de formas adequadas de manejo e estratégias de prevenção, delimitadas através das características dos indivíduos e necessidades regionais. Assim, este estudo teve por objetivo avaliar a prevalência da história de abuso físico e exposição à violência parental na infância segundo características sócio-demográficas, apresentando a magnitude e distribuição da violência em uma amostra representativa da população brasileira.

\section{Método}

\section{Procedimento de amostragem e coleta de dados}

O estudo atual analisa dados vindos do $I$ Levantamento Nacional Sobre os Padrões de Consumo de Álcool na População Brasileira 32, conduzido pela Universidade Federal de São Paulo (UNIFESP), Unidade de Estudo de Álcool e Outras Drogas (UNIAD).

A metodologia de amostragem foi desenhada para ter abrangência de todo o território nacional, representando toda a população brasileira de 14 anos de idade ou mais, de ambos os sexos e sem exclusão de qualquer parte do território nacional, inclusive áreas rurais. Não foram incluídas na amostra populações indígenas que vivem em aldeias, bem como residentes em território brasileiro que não falam a língua portuguesa, deficientes mentais e outros deficientes incapacitados de responder ao questionário. O desenho de amostragem seguiu padrões de estudos probabilísticos similares aos realizados nos Estados Unidos e na Europa e também aos estudos mais rigorosos feitos pelo Instituto Brasileiro de Geografia e Estatística (IBGE) 33 .

Entre novembro de 2005 e abril de 2006 foram realizadas 3.007 entrevistas da pesquisa. Foram selecionados 143 municípios brasileiros totalizando 325 setores censitários, utilizando a metodologia de amostra probabilística estratificada em três estágios. (a) Estágio 1: sorteio sistemático dos municípios, selecionados probabilisticamente pelo método PPT (Probabilidade 
Proporcional ao Tamanho), sendo a população de cada município tomada como base para tal seleção; (b) Estágio 2: seleção probabilística dos setores censitários também pelo mesmo método (PPT); (c) Estágio 3: contagem e sorteio aleatório simples dos domicílios, seguida pela seleção de um membro da casa a ser entrevistado, usando "a técnica do próximo aniversariante", a contar da data do arrolamento do domicílio.

A amostra foi composta por 2.522 entrevistas com participantes de 14 anos de idade ou mais, e uma cota extra de 485 entrevistas, estas últimas exclusivas para a população entre 14 e 17 anos de idade, totalizando 3.007 entrevistas. Esta cota extra se justifica pelo fato de que $10 \%$ da população brasileira se encontra nesta faixa etária. Uma hora de entrevista face-a-face foi conduzida na casa dos respondentes, por entrevistadores treinados que usaram um questionário impresso, fechado e padronizado.

A taxa de resposta obtida foi de $66 \%$. Em razão dos poucos estudos com este método realizados no Brasil, considerou-se como parâmetro a taxa de resposta estimada pela Pesquisa Social Brasileira (PESB) um outro estudo probabilístico feito no Brasil (para maiores informações sobre a PESB, ver a página na Internet: http:/ / www.uff. br/datauff/PESB.htm). Como a taxa de resposta da PESB foi de $68 \%$, também seguiu-se todo o procedimento de campo utilizado naquele estudo (abordagem, número de voltas, etc.). Assim, a taxa de resposta de $66 \%$ foi considerada satisfatória, visto que em pesquisas nessa área realizadas em países desenvolvidos como os Estados Unidos, os índices de não resposta em torno de $30 \%$ são aceitáveis 34 . Portanto, os dados obtidos são bastante confiáveis no sentido de serem representativos da população brasileira. Para uma discussão detalhada da metodologia do trabalho de campo utilizada no referido levantamento ver Zaleski et al. 35 .

\section{Mensurações}

O instrumento utilizado para coleta de dados do I Levantamento Nacional Sobre os Padrões de Consumo de Álcool na População Brasileira 32 foi baseado em uma versão do questionário Hispanic Americans Alcohol Survey - HABLAS 36, desenvolvido para estudos epidemiológicos sobre padrões de consumo de álcool em hispano-americanos. O questionário foi traduzido e adaptado para a população brasileira através de estudos pilotos qualitativos e quantitativos para avaliar a aplicabilidade e aceitabilidade na população brasileira. A versão completa do questionário poderá ser encontrada nos sites da UNIAD (http://www.uniad.org.br) e da Secretaria Nacio- nal Antidrogas - SENAD (http://www.senad.gov. br). Para o presente estudo, foram utilizados os dados da seção referente às variáveis sócio-demográficas e a seção referente ao histórico de violência na infância. Os itens do HABLAS para avaliação do abuso físico na infância foram adaptados da Conflict Tactics Scales (CTS), Form $R 37$ e Revised Conflict Tactics Scales (CTS2) 38. Desta forma, neste trabalho, o termo abuso foi restringido a abuso físico intra-familiar caracterizado pelo uso da força física de pais e/ou cuidadores contra crianças e adolescentes e violência parental também caracterizada pelo uso da força física com a intenção de provocar danos ao parceiro íntimo, de acordo com os critérios de Straus \& Gelles 39 .

\section{- Abuso físico na infância}

Os respondentes foram questionados se durante sua infância ou adolescência seus pais ou as pessoas que os criaram tinham feito, alguma vez, uma das seguintes coisas: bateu com alguma coisa, bateu em você, queimou ou escaldou, ameaçou com uma faca ou uma arma, ou usou uma faca ou uma arma. De acordo com suas respostas, os indivíduos foram alocados em um de três grupos: sem história de abuso físico na infância, abuso físico moderado (bateram em você), ou abuso físico severo na infância (bateu em você com alguma coisa, queimou ou escaldou, ameaçou com uma faca ou uma arma, ou usou uma faca ou uma arma).

\section{- Exposição à violência parental na infância}

Os respondentes foram questionados se durante sua infância ou adolescência tinham observado seus pais ou as pessoas que os criaram ameaçarem um ao outro de praticarem violência física, ou serem realmente violentos fisicamente um com o outro. Com base nas suas respostas, os indivíduos foram alocados em três grupos: sem exposição à violência parental (nunca observaram ameaça de violência ou violência física parental), exposição à violência parental moderada (testemunharam ameaça de agressão parental) e exposição à violência parental severa (testemunhou ameaça e agressão física parental, juntas).

\section{- Variáveis sócio-demográficas}

a) Sexo: homem ou mulher;

b) Idade: mensurada continuamente em anos e codificada em oito categorias: 14-19; 20-29; 30-39; 40-49; 50-59; 60-69; 70-79; 80 ou mais;

c) Raça: auto-identificada com relação à cor da pele em brancos, negros, pardos ou outros; 
d) Estado civil: classificado como solteiros, casados ou com companheiro, viúvos, separados ou desquitados, e divorciados;

e) Nível educacional: classificação de acordo com o nível mais alto ou ano escolar completado e codificado em 4 categorias: analfabeto a $4^{\mathrm{a}}$ série do ensino fundamental; $5^{\mathrm{a}}$ a $8^{\mathrm{a}}$ série do ensino fundamental; ensino médio; e ensino superior;

f) Classe social: avaliada segundo os critérios de classificação econômica do Brasil que são um instrumento que possibilita de forma confiável estabelecer parâmetros de renda familiar de cada classe social, de A a E;

g) Renda familiar: identificada conforme o valor total da renda familiar mensal, na seguinte escala: até R\$ 450,00; R\$ 451,00 a R\$ 750,00; R\$ 751,00 a $\mathrm{R} \$ 1.200,00 ; \mathrm{R} \$ 1.201,00$ a $\mathrm{R} \$ 2.500,00$; mais de R\$ 2.500,00;

h) Situação de trabalho: classificada em duas categorias: desempregado (desempregado, dona de casa que não trabalha, aposentado(a), estudante que não trabalha) e empregado (trabalha formalmente, trabalha mesmo sem carteira, trabalha como aprendiz, ajudante, e outros, trabalhou ou tentou trabalhar na última semana);

i) Região do país: conforme a moradia: Norte, Nordeste, Centro-Oeste, Sudeste e Sul.

\section{Considerações éticas}

O estudo foi aprovado pelo Comitê de Ética em Pesquisa da Escola Paulista de Medicina (EPM) da UNIFESP (CEP n ${ }^{\circ}$. 1.672/04) e contemplou todos os aspectos éticos previstos na Resolução $n^{\circ}$. 196/96 do Conselho Nacional de Saúde do Ministério da Saúde.

\section{Análise estatística}

As análises foram realizadas através do programa SPSS 13.0 (SPSS Inc., Chicago, Estados Unidos). Realizou-se análise descritiva para a caracterização da amostra e da distribuição do histórico de violência na infância. Em seguida, para se estabelecer associação entre ocorrência de história de abuso físico e exposição à violência parental na infância com as variáveis sócio-demográficas foram utilizados testes de homogeneidade baseados na estatística de qui-quadrado de Pearson, levando-se em consideração a estrutura complexa do plano amostral sendo utilizado o módulo complex samples do SPSS. Admitiu-se um nível de significância estatística de 5\%.

\section{Resultados}

\section{Características da amostra estudada}

A amostra estudada incluiu $52,10 \%$ mulheres ( $n=1.722)$ e $47,9 \%$ homens $(n=1.285)$, com idade média de 35,7 anos (variando de 14 a 91 anos), $50,5 \%$ (1.466) dos sujeitos eram de cor da pele branca, e $55,4 \%$ eram casados ou viviam com um(a) companheiro(a). Aproximadamente 34\% dos sujeitos $(\mathrm{n}=1.017)$ apresentavam baixos níveis de instrução, sendo que destes, 23\% nunca freqüentaram a escola ou tinham apenas as séries iniciais do ensino fundamental completas. Com relação à situação de trabalho, 56,6\% ( $\mathrm{n}=1.702)$ dos sujeitos relataram trabalhar com carteira assinada. Para a variável nível sócio-econômico as maiores concentrações na amostra foram nas classes D e C, (39\% ou 1.173 sujeitos e $34 \%$ ou 1.024 sujeitos, respectivamente), seguidas pela classe B (15,4\% ou 462 sujeitos) e $36 \%$ dos sujeitos relatam uma renda familiar mensal de até $\mathrm{R} \$ 450,00$. A amostra foi constituída por sujeitos de todas as regiões do país, desta forma, nas análises a seguir os resultados são apresentados com expansão de modo a possibilitar uma representatividade nacional.

\section{Violência física na infância segundo características sócio-demográficas}

As características sócio-demográficas segundo a história de abuso físico na infância são apresentadas na Tabela 1. Na amostra estudada, a prevalência de uma história de abuso físico na infância foi de 44,1\% ( $n=1.323$ ), sendo que 33,8\% ( $\mathrm{n}=1.014$ ) relataram uma história de abuso físico moderado e $10,3 \%(n=309)$ relataram abuso físico severo.

As comparações entre sujeitos sem história de abuso físico e aqueles que relataram algum tipo de abuso físico na infância revelam que os grupos foram estatisticamente equivalentes quanto ao gênero, faixa etária, cor da pele, estado civil, classe sócio-econômica, nível de instrução e região do país, mesmo considerando a severidade do abuso físico sofrido. Diferenças estatisticamente significativas foram encontradas apenas com relação à situação de trabalho, entretanto a renda familiar não difere significativamente através dos três grupos.

Os participantes com história de abuso físico apresentaram também mais freqüentemente história positiva para exposição à violência parental em relação àqueles sem história de abuso físico.

As análises de abuso físico na infância e exposição à violência parental combinadas demons- 
Distribuição dos respondentes segundo histórico de violência na infância, por características sócio-demográficas, em percentual.

\begin{tabular}{|c|c|c|c|c|c|c|}
\hline \multirow[t]{2}{*}{ Características } & \multicolumn{5}{|c|}{ Violência na infância } & \multirow[t]{2}{*}{$\mathrm{n}$} \\
\hline & $\begin{array}{l}\text { Sem história de } \\
\text { abuso físico } \\
\%(I C 95 \%)\end{array}$ & $\begin{array}{l}\text { Abuso físico } \\
\text { moderado } \\
\% \text { (IC95\%) }\end{array}$ & $\begin{array}{l}\text { Abuso físico } \\
\text { severo } \\
\% \text { (IC95\%) }\end{array}$ & $\chi^{2}$ & Valor de p & \\
\hline Total & $55,9(52,40-9,30)$ & $33,8(30,80-37,00)$ & $10,3(8,80-12,00)$ & - & - & 2.999 \\
\hline Sexo & & & & 4,96 & 0,240 & \\
\hline Masculino & $54,5(50,10-58,90)$ & $35,8(31,80-40,00)$ & $9,7(7,60-12,40)$ & & & 1.282 \\
\hline Feminino & $57,1(53,40-60,80)$ & $32,0(28,70-35,60)$ & $10,8(9,00-13,10)$ & & & 1.717 \\
\hline Faixa etária (anos) & & & & 26,90 & 0,162 & \\
\hline $14-19$ & $53,2(46,70-59,60)$ & $36,9(31,10-43,10)$ & $9,9(7,00-13,80)$ & & & 759 \\
\hline $20-29$ & $52,0(46,50-57,50)$ & $36,2(31,40-41,30)$ & $11,8(8,70-15,70)$ & & & 584 \\
\hline $30-39$ & $58,2(52,60-63,50)$ & $32,3(27,50-37,40)$ & $9,6(7,00-13,00)$ & & & 547 \\
\hline $40-49$ & $54,9(48,80-60,80)$ & $33,9(28,50-39,80)$ & $11,2(7,90-15,80)$ & & & 417 \\
\hline $50-59$ & $56,6(49,30-63,60)$ & $32,6(26,10-39,90)$ & $10,8(7,70-14,90)$ & & & 294 \\
\hline $60-69$ & $58,3(50,50-65,80)$ & $33,6(26,80-41,10)$ & $8,1(5,30-12,20)$ & & & 244 \\
\hline $70-79$ & $71,4(61,40-79,60)$ & $22,5(15,00-32,30)$ & $6,1(2,40-14,80)$ & & & 120 \\
\hline 80 ou mais & $77,9(60,30-89,10)$ & $14,4(5,90-31,30)$ & $7,6(2,40-21,90)$ & & & 34 \\
\hline Cor da pele & & & & 19,24 & 0,089 & \\
\hline Branca & $59,0(54,80-63,10)$ & $32,0(28,50-35,80)$ & $9,0(7,10-11,20)$ & & & 1.463 \\
\hline Preta & $47,8(40,90-54,80)$ & $39,4(33,10-45,90)$ & $12,9(9,00-18,10)$ & & & 325 \\
\hline Parda & $54,6(49,20-59,80)$ & $34,3(29,60-39,30)$ & $11,2(8,70-14,30)$ & & & 1.128 \\
\hline Outras & $48,8(36,00-61,90)$ & $37,9(25,60-51,90)$ & $13,3(6,60-24,80)$ & & & 83 \\
\hline Instrução & & & & 14,76 & 0,249 & \\
\hline Analfabeto/4a série do Ensino Fundamental & $59,2(54,50-63,80)$ & $31,7(27,50-36,20)$ & $9,1(7,20-11,40)$ & & & 1.012 \\
\hline 5ạ a 8a série do Ensino Fundamental & $53,6(48,70-58,30)$ & $34,3(29,90-39,10)$ & $12,1(9,50-15,30)$ & & & 872 \\
\hline Ensino Médio & $56,2(51,30-60,90)$ & $34,8(30,50-39,40)$ & $9,0(6,90-11,80)$ & & & 948 \\
\hline Ensino Superior & $49,9(40,70-59,20)$ & $37,1(28,10-47,00)$ & $13,0(7,50-21,50)$ & & & 167 \\
\hline Estado civil & & & & 18,80 & 0,075 & \\
\hline Solteiro & $56,1(50,80-61,20)$ & $32,8(28,10-37,90)$ & $11,1(8,60-14,20)$ & & & 1.153 \\
\hline Casado/ou com companheiro(a) & $54,5(50,70-58,20)$ & $36,1(32,70-39,70)$ & $9,4(7,90-11,30)$ & & & 1.442 \\
\hline Viúvo & $64,0(55,80-71,50)$ & $26,5(19,80-34,50)$ & $9,5(5,80-15,10)$ & & & 200 \\
\hline Desquitado/Divorciado & $59,4(45,70-71,80)$ & $26,8(16,10-41,30)$ & $13,8(7,20-24,60)$ & & & 76 \\
\hline Separado & $61,1(50,20-71,00)$ & $23,2(15,60-33,10)$ & $15,7(9,30-25,30)$ & & & 128 \\
\hline Situação de trabalho & & & & 11,82 & 0,022 & \\
\hline Não & $59,8(55,50-64,00)$ & $30,9(27,10-35,00)$ & $9,3(7,30-11,70)$ & & & 1.345 \\
\hline Sim & $53,4(49,50-57,30)$ & $35,7(32,20-39,30)$ & $10,9(9,10-13,10)$ & & & 1.654 \\
\hline Faixas de renda familiar & & & & 21,20 & 0,213 & \\
\hline Até $\mathrm{R} \$ 450,00$ & $53,8(48,50-59,00)$ & $34,8(30,30-39,70)$ & $11,4(9,00-14,50)$ & & & 1.230 \\
\hline $\mathrm{R} \$ 451,00$ a $\mathrm{R} \$ 750,00$ & $61,2(55,60-66,40)$ & $31,5(26,60-36,80)$ & $7,4(5,40-9,90)$ & & & 584 \\
\hline $\mathrm{R} \$ 751,00$ a $\mathrm{R} \$ 1.200,00$ & $52,3(46,20-58,30)$ & $35,5(29,40-42,10)$ & $12,2(9,10-16,20)$ & & & 482 \\
\hline $\mathrm{R} \$ 1.201,00$ a $\mathrm{R} \$ 2.500,00$ & $49,0(41,50-56,50)$ & $39,9(33,10-47,20)$ & $11,1(7,40-16,30)$ & & & 303 \\
\hline Mais de $\mathrm{R} \$ 2.500,00$ & $57,7(46,10-68,40)$ & $30,6(20,50-42,80)$ & $11,8(5,70-22,70)$ & & & 120 \\
\hline
\end{tabular}

(continua) 
Tabela 1 (continuação)

\begin{tabular}{|c|c|c|c|c|c|c|}
\hline \multirow[t]{2}{*}{ Características } & \multicolumn{5}{|c|}{ Violência na infância } & \multirow[t]{2}{*}{$\mathbf{n}$} \\
\hline & $\begin{array}{l}\text { Sem história de } \\
\text { abuso físico } \\
\%(I C 95 \%)\end{array}$ & $\begin{array}{l}\text { Abuso físico } \\
\text { moderado } \\
\% \text { (IC95\%) }\end{array}$ & $\begin{array}{c}\text { Abuso físico } \\
\text { severo } \\
\% \text { (IC95\%) }\end{array}$ & $\chi^{2}$ & Valor de $p$ & \\
\hline Classe sócio-econômica & & & & 10,89 & 0,659 & \\
\hline A & $53,7(36,90-69,60)$ & $42,1(26,30-59,80)$ & $4,2(0,90-17,40)$ & & & 65 \\
\hline B & $54,2(47,10-61,20)$ & $34,6(28,20-41,60)$ & $11,1(7,40-16,40)$ & & & 389 \\
\hline C & $56,1(51,80-60,20)$ & $33,3(29,60-37,10)$ & $10,7(8,80-13,00)$ & & & 974 \\
\hline $\mathrm{D}$ & $57,7(52,90-62,30)$ & $32,2(28,00-36,80)$ & $10,1(7,80-13,00)$ & & & 1.256 \\
\hline$E$ & $50,7(41,90-59,30)$ & $39,1(31,30-47,50)$ & $10,2(6,70-15,30)$ & & & 315 \\
\hline Região do país & & & & 38,33 & 0,149 & \\
\hline Norte & $48,0(34,30-62,10)$ & $36,5(25,40-49,10)$ & $15,5(7,90-28,30)$ & & & 208 \\
\hline Centro-Oeste & $61,2(48,50-72,60)$ & $27,2(16,70-41,00)$ & $11,5(7,30-17,80)$ & & & 236 \\
\hline Nordeste & $55,4(48,40-62,30)$ & $35,1(28,90-41,70)$ & $9,5(6,80-13,10)$ & & & 882 \\
\hline Sudeste & $59,6(54,80-64,30)$ & $30,5(26,40-34,90)$ & $9,9(8,10-12,10)$ & & & 1.272 \\
\hline Sul & $47,6(38,60-56,70)$ & $42,8(34,20-51,80)$ & $9,6(6,80-13,50)$ & & & 401 \\
\hline Exposição & & & & 332,91 & $<0,001$ & \\
\hline Sem exposição à violência parental & $63,1(59,20-66,80)$ & $31,8(28,50-35,40)$ & $5,1(3,80-6,70)$ & & & 2.184 \\
\hline Exposição à violência parental moderada & $36,8(28,60-45,90)$ & $45,7(36,10-55,70)$ & $17,4(11,10-26,40)$ & & & 214 \\
\hline Exposição à violência parental severa & $35,3(30,50-40,40)$ & $36,4(31,80-41,20)$ & $28,3(24,20-32,80)$ & & & 588 \\
\hline
\end{tabular}

IC95\%: intervalo de 95\% de confiança.

traram que 5,1\% dos indivíduos sem exposição à violência parental sofreram abuso físico severo na infância, e entre aqueles expostos à violência parental moderada na infância esta porcentagem aumenta para $17,4 \%$. Já entre os expostos à violência parental severa, $28,3 \%$ sofreram abuso físico severo na infância. Também, pode-se observar que histórias de abuso físico moderado são mais comuns nos sujeitos expostos à violência parental moderada $(45,7 \%$, enquanto que nas demais categorias de exposição este percentual não é superior a 36,4\%). Desta forma, observa-se que quanto maior o grau de exposição à violência parental, maior a porcentagem de abuso físico severo sofrido na infância.

\section{Exposição à violência parental na infância segundo características sócio-demográficas}

Na Tabela 2 são apresentadas as características sócio-demográficas segundo história de exposição à violência parental. A prevalência de exposição à violência parental na infância foi de $26,1 \%$ $(\mathrm{n}=781)$. Entre aqueles que relatam tal exposição, $7,5 \%(n=224)$ testemunharam violência parental moderada e $18,6 \%(n=557)$ testemunharam violência parental severa.

As análises demonstraram que existiram associações significativas entre exposição à violên- cia parental na infância e sexo $(p=0,002)$, faixa etária $(\mathrm{p}=0,002)$, classe sócio-econômica $(\mathrm{p}=$ $0,011)$, região $(p=0,022)$ e instrução $(p=0,006)$. Mulheres foram mais sujeitas $(21,2 \%)$ à exposição mais severa do que os homens (15,8\%). A prevalência da exposição à violência parental mais severa declinou com a idade dos respondentes. Com relação à classe social, nota-se que na classe C, 22,7\% dos sujeitos tiveram exposição severa, porcentagem maior que nas demais classes.

A exposição à violência parental moderada foi mais prevalente na Região Norte. As Regiões Centro-Oeste e Sul do Brasil apresentaram maiores porcentagens de exposição à violência parental severa, 25,9\% e 23,9\%, respectivamente. Analisando o nível de instrução com a história de exposição à violência parental verificou-se que é mais provável que os sujeitos sem história de exposição também possuam maior nível de instrução $(p=0,006)$ do que sujeitos expostos à violência parental. Dos que possuem até o ensino fundamental, $23 \%$ testemunharam violência parental severa e esta porcentagem cai gradativamente à medida que a instrução aumenta, chegando ao patamar de $13 \%$ em indivíduos com instrução superior.

Não houve nenhuma diferença significativa entre os grupos para estado civil, renda familiar, situação de trabalho e cor da pele. 
Distribuição dos respondentes segundo exposição à violência parental por características sócio-demográficas, em percentual.

\begin{tabular}{|c|c|c|c|c|c|c|}
\hline \multirow[t]{2}{*}{ Características } & \multicolumn{5}{|c|}{ Violência na infância } & \multirow[t]{2}{*}{$\mathrm{n}$} \\
\hline & $\begin{array}{l}\text { Sem exposição à } \\
\text { violência parental } \\
\% \text { (IC95\%) }\end{array}$ & $\begin{array}{l}\text { Exposiçãa à } \\
\text { violência parental } \\
\text { moderada } \\
\% \text { (IC95\%) }\end{array}$ & $\begin{array}{c}\text { Exposição à } \\
\text { violência parental } \\
\text { severa } \\
\% \text { (IC95\%) }\end{array}$ & $\chi^{2}$ & Valor de $p$ & \\
\hline Total & $73,9(71,60-76,0)$ & $7,5(6,30-8,90)$ & $18,6(16,70-20,70)$ & - & - & 2.993 \\
\hline Sexo & & & & 16,12 & 0,002 & \\
\hline Masculino & $75,9(72,8-78,8)$ & $8,3(6,60-10,50)$ & $15,8(13,50-18,40)$ & & & 1.276 \\
\hline Feminino & $72,0(69,2-74,7)$ & $6,7(5,50-8,20)$ & $21,2(18,90-23,80)$ & & & 1.717 \\
\hline Faixa etária (anos) & & & & 47,75 & 0,002 & \\
\hline $14-19$ & $74,0(69,30-78,10)$ & $6,8(5,10-9,20)$ & $19,2(15,70-23,20)$ & & & 758 \\
\hline $20-29$ & $67,9(62,40-72,90)$ & $8,3(5,70-11,90)$ & $23,8(19,40-28,90)$ & & & 582 \\
\hline $30-39$ & $72,2(67,40-76,60)$ & $10,2(7,40-13,80)$ & $17,6(14,20-21,70)$ & & & 544 \\
\hline $40-49$ & $76,6(71,80-80,90)$ & $4,5(2,90-6,90)$ & $18,8(14,80-23,60)$ & & & 415 \\
\hline $50-59$ & $77,4(70,90-82,70)$ & $6,1(3,40-10,50)$ & $16,6(11,90-22,70)$ & & & 295 \\
\hline $60-69$ & $78,4(72,30-83,60)$ & $10,0(6,60-14,80)$ & $11,6(7,80-16,90)$ & & & 245 \\
\hline $70-79$ & $85,4(77,10-80,90)$ & $4,3(1,40-12,70)$ & $10,2(5,70-17,60)$ & & & 121 \\
\hline 80 ou mais & $83,0(66,10-92,40)$ & $4,7(1,40-13,90)$ & $12,4(4,20-31,40)$ & & & 33 \\
\hline Cor da pele & & & & 14,61 & 0,169 & \\
\hline Branca & $74,0(70,90-76,80)$ & $8,1(6,30-10,30)$ & $18,0(15,50-20,70)$ & & & 1.461 \\
\hline Preta & $69,6(62,30-76,00)$ & $5,4(3,40-8,40)$ & $25,0(19,10-32,10)$ & & & 324 \\
\hline Parda & $75,6(72,00-79,00)$ & $7,1(5,30-9,60)$ & $17,2(14,70-20,10)$ & & & 1.125 \\
\hline Outras & $68,6(56,00-78,90)$ & $10,3(4,40-22,30)$ & $21,2(13,00-32,50)$ & & & 83 \\
\hline Instrução & & & & 38,40 & 0,006 & \\
\hline Analfabeto/4 ${ }^{a}$ série do Ensino Fundamental & $78,3(75,00-81,30)$ & $7,0(5,10-9,50)$ & $14,7(12,30-17,60)$ & & & 1.014 \\
\hline $5^{\mathrm{a}}$ a $8^{\mathrm{a}}$ série do Ensino Fundamental & $71,4(67,60-74,80)$ & $5,7(4,20-7,60)$ & $23,0(19,70-26,70)$ & & & 864 \\
\hline Ensino Médio & $69,8(65,90-73,50)$ & $9,5(7,30-12,20)$ & $20,7(17,70-24,10)$ & & & 947 \\
\hline Ensino Superior & $77,6(68,10-85,00)$ & $9,0(4,60-16,90)$ & $13,3(7,40-22,80)$ & & & 168 \\
\hline Estado civil & & & & 9,02 & 0,508 & \\
\hline Solteiro & $73,9(70,10-77,30)$ & $8,2(6,10-10,80)$ & $18,0(15,0-21,30)$ & & & 1.153 \\
\hline Casado ou com companheiro(a) & $74,0(71,10-76,60)$ & $7,2(5,80-9,00)$ & $18,8(16,40-21,50)$ & & & 1.438 \\
\hline Viúvo & $78,9(71,20-85,00)$ & $5,9(2,90-11,50)$ & $15,3(10,20-22,20)$ & & & 199 \\
\hline Desquitado/Divorciado & $68,0(54,40-79,20)$ & $4,1(1,30-12,50)$ & $27,9(17,20-41,90)$ & & & 74 \\
\hline Separado & $68,9(58,90-77,40)$ & $10,0(5,20-18,40)$ & $21,2(14,10-30,40)$ & & & 129 \\
\hline Situação de Trabalho & & & & 0,87 & 0,735 & \\
\hline Não & $74,7(71,70-77,50)$ & $7,0(5,40-9,00)$ & $18,3(16,00-20,80)$ & & & 1.342 \\
\hline Sim & $73,4(70,30-76,20)$ & $7,8(6,30-9,60)$ & $18,8(16,30-21,60)$ & & & 1.651 \\
\hline Faixas de renda familiar & & & & 19,15 & 0,285 & \\
\hline Até $\mathrm{R} \$ 450,00$ & $73,6(69,80-77,10)$ & $6,5(4,70-8,90)$ & $19,9(16,90-23,30)$ & & & 1.226 \\
\hline $\mathrm{R} \$ 451,00$ a $\mathrm{R} \$ 750,00$ & $75,5(70,50-79,90)$ & $6,3(4,10-9,60)$ & $18,2(14,70-22,30)$ & & & 584 \\
\hline $\mathrm{R} \$ 751,00$ a $\mathrm{R} \$ 1.200,00$ & $72,4(67,40-76,90)$ & $11,4(8,00-15,90)$ & $16,2(12,70-20,50)$ & & & 482 \\
\hline $\mathrm{R} \$ 1.201,00$ a $\mathrm{R} \$ 2.500,00$ & $70,8(63,50-77,20)$ & $7,3(4,50-11,50)$ & $21,9(16,50-28,50)$ & & & 302 \\
\hline Mais de $R \$ 2.500,00$ & $70,3(57,60-80,50)$ & $9,4(4,70-17,90)$ & $20,3(11,40-33,50)$ & & & 120 \\
\hline
\end{tabular}

(continua) 
Tabela 2 (continuação)

\begin{tabular}{|c|c|c|c|c|c|c|}
\hline \multirow[t]{2}{*}{ Características } & \multicolumn{5}{|c|}{ Violência na infância } & \multirow[t]{2}{*}{$n$} \\
\hline & $\begin{array}{c}\text { Sem exposição à } \\
\text { violência parental } \\
\%(I C 95 \%)\end{array}$ & $\begin{array}{c}\text { Exposição à } \\
\text { violência parental } \\
\text { moderada } \\
\%(\text { (IC95\%) }\end{array}$ & $\begin{array}{c}\text { Exposição à } \\
\text { violência parental } \\
\text { severa } \\
\%(\text { IC95\%) }\end{array}$ & $\chi^{2}$ & Valor de $\mathrm{p}$ & \\
\hline Classe sócio-econômica & & & & 35,97 & 0,011 & \\
\hline$A$ & $81,6(66,60-90,80)$ & $7,2(2,90-16,80)$ & $11,2(4,00-27,80)$ & & & 65 \\
\hline B & $75,6(69,30-81,00)$ & $10,0(6,60-14,90)$ & $14,3(10,20-19,90)$ & & & 390 \\
\hline C & $68,6(64,70-72,10)$ & $8,7(6,80-11,10)$ & $22,7(19,70-26,10)$ & & & 968 \\
\hline $\mathrm{D}$ & $76,1(72,90-79,00)$ & $5,9(4,60-7,60)$ & $18,0(15,50-20,80)$ & & & 1.254 \\
\hline$E$ & $79,3(72,80-84,50)$ & $5,4(2,60-10,60)$ & $15,4(10,80-21,40)$ & & & 316 \\
\hline Região do país & & & & 53,79 & 0,002 & \\
\hline Norte & $75,8(63,80-84,80)$ & $11,8(5,80-22,60)$ & $12,4(7,20-20,50)$ & & & 203 \\
\hline Centro-Oeste & $65,9(59,60-71,70)$ & $8,2(4,70-13,80)$ & $25,9(18,20-35,40)$ & & & 235 \\
\hline Nordeste & $80,9(76,50-84,70)$ & $5,6(3,70-8,40)$ & $13,5(10,60-17,10)$ & & & 883 \\
\hline Sudeste & $73,0(69,80-76,00)$ & $7,0(5,50-8,80)$ & $20,0(17,20-23,10)$ & & & 1.270 \\
\hline Sul & $66,3(59,70-72,30)$ & $9,80(6,90-13,80)$ & $23,9(18,20-30,80)$ & & & 402 \\
\hline Abuso físico na infância & & & & 332,91 & $<0,001$ & \\
\hline Sem história de abuso físico & $83,3(80,90-85,50)$ & $4,90(3,70-6,50)$ & $11,8(10,00-13,80)$ & & & 1.662 \\
\hline Abuso físico moderado & $69,7(66,00-73,20)$ & $10,10(7,80-13,10)$ & $20,1(17,00-23,70)$ & & & 1.026 \\
\hline Abuso físico severo & $36,4(29,10-44,50)$ & $12,60(8,00-19,20)$ & $51,0(43,10-58,80)$ & & & 298 \\
\hline
\end{tabular}

IC95\%: intervalo de 95\% de confiança.

As análises da exposição à violência parental e abuso físico na infância combinadas demonstraram uma associação significativa entre estes dois tipos de violência da infância $(p<0,001)$, sendo que dos sujeitos que sofreram abuso físico severo, $51 \%$ sofreram também exposição à violência parental severa.

\section{Discussão}

A prevalência de algum tipo de abuso físico na infância relatado pelos sujeitos desse estudo foi de $44,1 \%$. Os resultados foram semelhantes aos encontrados em uma pesquisa com amostra nacionalmente representativa de homens e mulheres adultos $(\mathrm{n}=16.000)$, avaliados pela CTS, oriunda do National Violence Against Women Survey 40, coletados entre 1995 a 1996, que encontrou uma prevalência de $47 \%$ de relato de experiência de abuso físico na infância.

Entretanto, comparada a outros estudos também internacionais, com amostras nacionalmente representativas, a prevalência de abuso físico na infância do presente estudo foi bem mais elevada do que as relatadas por Briere \& Elliott 16 , os quais encontraram que $21 \%$ de americanos adultos $(\mathrm{n}=1.442)$ tinham história de abuso fí- sico na infância, sendo que os sujeitos somente foram considerados nesta condição se antes dos 18 anos de idade seus pais ou cuidadores fizeram uma das seguintes coisas: bateram com o punho fechado, chutaram, jogaram no chão ou em uma parede ou escada abaixo; fizeram alguma coisa com a finalidade de deixar marcas, contusões, queimaduras, sangramento, perda dos dentes ou ossos quebrados. Hussey et al. 5 também encontraram um percentual de $28,4 \%$ dos adultos $(\mathrm{n}=15.197)$ avaliados entre 2001 e 2002 que relataram abuso físico na infância, de modo que o abuso físico foi investigado questionando aos participantes se durante a infância seus pais ou cuidadores tinham praticado algum dos seguintes atos: bater, chutar ou esbofetear.

Utilizando a mesma abordagem do estudo atual para avaliação do histórico de violência na infância, Caetano et al. ${ }^{41}$ encontraram uma prevalência de abuso físico na infância de aproximadamente $60 \%$ em uma amostra nacionalmente representativa de casais americanos $(n=2.880)$.

Com relação à comparação por tipo e severidade do abuso físico, os resultados encontrados foram semelhantes aos resultados relatados no National Family Violence Survey, realizado em 1985, que investigou a violência contra criança em famílias que tinham pelo menos um filho 
com menos de 18 anos, tendo encontrado que aproximadamente $10 \%$ dos sujeitos $(n=3.520$ famílias) relataram agressão física severa por um cuidador 39 . Em outra análise vinda do mesmo estudo com uma sub-amostra ( $\mathrm{n}=3.232$ ) Wolfner \& Gelles 42 verificaram uma prevalência de $62 \%$ de violência moderada (empurrar, agarrar, esbofetear ou espancar) e 11\% de violência severa (chutar, bater, entre outras).

Apesar do grande desenvolvimento de pesquisas sobre violência doméstica nos últimos anos, é escassa a literatura brasileira sobre dados nacionalmente representativos de prevalência de abuso físico na infância relatado pela população adulta. Outros estudos realizados nessa área, devido às limitações de suas amostras a determinadas faixas etárias dificultam comparações diretas com o estudo atual. No entanto, face às suas relevâncias, tais estudos podem servir para ilustrar a importância do tema. Porém, estes estudos utilizam-se de diferentes instrumentos para mensurar a violência, além de referirem-se a cortes temporais variados.

Dentre estes estudos destacam-se a pesquisa realizada por Assis \& Avanci 43, um levantamento epidemiológico realizado em 2002, no qual examinaram estudantes de escolas públicas e particulares ( $n=1.685)$ no Município de São Gonçalo, Rio de Janeiro, e encontraram uma alta prevalência de relato para violência familiar, sendo que $14,6 \%$ sofreram violência física severa desferida pelo pai ou mãe, confirmando a alta prevalência de violência familiar.

Também destaca-se o estudo realizado por Bordin et al. 7 com mães $(n=89)$ selecionadas aleatoriamente em uma comunidade de baixa renda em Embu, na cidade de São Paulo, o qual encontrou percentuais semelhantes às do presente estudo para punição física severa dos filhos $(10,1 \%)$ e para punição física não grave $(75,3 \%)$. As autoras consideraram punição física severa atos como sacudir/chacoalhar (se menores de dois anos), chutar, esganar, sufocar, queimar, espancar ou ameaçar com arma. Para avaliação de punição física não grave considerou atos como bater nas nádegas, puxar a orelha, entre outros. As autoras concluíram ser freqüente na comunidade estudada o castigo físico severo em crianças e adolescentes pela mãe e/ou maridos, ou parceiros.

O estudo atual também encontrou resultados semelhantes aos encontrados em estudos de amostras americanas nacionalmente representativas que sugerem que entre $13 \%$ a $20 \%$ dos adultos recordam testemunhar o conflito físico entre os pais na infância 4,17,31.

Com relação ao tipo de exposição à violência parental, Caetano et al. ${ }^{41}$ encontraram uma pre- valência de $10 \%$ para aqueles que testemunharam alguma ameaça de agressão física parental e $31 \%$ para os que testemunharam ameaça e agressão física parental na infância, em uma amostra nacionalmente representativa de casais americanos. Estes resultados também se assemelham aos encontrados no estudo atual.

Similarmente às literaturas prévias, o estudo atual encontrou que é mais provável que os sujeitos expostos à violência parental na infância também tenham experimentado abuso físico na infância. A co-ocorrência de exposição à violência parental e abuso físico na infância foi consistente novamente com os resultados obtidos por Caetano et al. 41 , em que $24 \%$ dos sujeitos com histórico de exposição à violência parental também relatavam abuso físico na infância.

As relações entre características sócio-demográficas e relatos de violência na infância variaram conforme o tipo de violência sofrida. $\mathrm{O}$ abuso físico na infância não foi associado a quase todas as características sócio-demográficas, com exceção da situação de trabalho. Uma possível explicação para este achado isolado foi a codificação desta variável em duas categorias para as análises bivariadas (empregado e desempregado). A falta de uniformidade dos resultados do presente estudo com a literatura prévia sugere que estas diferenças podem ser explicadas, em parte, por diferentes estratégias metodológicas utilizadas para avaliação do histórico do abuso. No estudo atual, a definição de abuso físico foi específica para eventos em que os sujeitos perceberam os referidos atos de agressão perpetrados pelos pais ou cuidadores. Assim, era mais provável que estes incidentes fossem mais severos e, conseqüentemente, menos prevalentes. Além disso, foi avaliado especificamente o histórico de abuso físico, não se levando em consideração outros tipos de abuso intra-familiar, tais como abuso psicológico, sexual e negligência parental, além de não haver sido avaliada a ocorrência de violência extra-familiar.

Os achados para a exposição à violência parental do estudo atual mostraram ser esta mais prevalente em sujeitos mais jovens, na faixa etária entre 14 e 29 anos. Estes resultados são semelhantes aos de Zaleski et al. ${ }^{29}$, que encontraram uma maior prevalência de violência íntima nesta mesma faixa etária, em uma amostra de 1.445 casais (631 homens e 814 mulheres), casados ou que co-habitavam, vindos da mesma amostra do presente estudo. Ademais, diversos estudos apontam para um aumento da violência em gerações mais recentes $1,2,39$.

Quanto à distribuição geográfica, foi encontrada uma maior prevalência de relato de abuso físico e exposição à violência parental moderada 
na região Norte, considerando que o resultado do abuso físico não foi estatisticamente significante, mas que houve uma prevalência ligeiramente maior nesta região. Já para a exposição à violência parental severa, percentuais mais altos foram encontrados nas regiões Centro-Oeste e Sul do país. As regiões Norte e Nordeste foram apontadas por Reichenheim et al. ${ }^{44}$ como as regiões de maior prevalência de violência íntima entre casais na comparação deste evento em 15 capitais brasileiras, incluindo o Distrito Federal. Entretanto, Zalescki et al. ${ }^{29}$ não encontraram diferenças de distribuição da prevalência da violência íntima na análise de casais vindos da mesma amostra do estudo atual, nas diferentes regiões do Brasil. Os estudos supracitados avaliaram a violência íntima entre casais, enquanto o estudo atual avaliou o histórico de violência na infância, o que pode explicar, em parte, as diferenças nos resultados obtidos para a distribuição geográfica da prevalência de violência nas diferentes regiões do Brasil.

A exposição à violência parental foi inversamente relacionada com o grau de instrução e a classe social. Diferenças raciais não foram relevantes tanto para histórico de abuso físico quanto para exposição à violência parental na infância. A não associação do histórico de abuso físico na infância com algumas características sócio-demográficas dos sujeitos podem estar associadas ao sub-relato da violência na infância. A literatura demonstra que sujeitos expostos à violência na infância tendem a desenvolver padrões de comportamentos agressivos na idade adulta $13,17,19$. Este fato, por si só, poderia distorcer a percepção do sujeito com relação a atos fisicamente violentos. Além disso, a falta de uniformidade dos resultados do presente estudo com a literatura prévia sugere que cada tipo de abuso pode estar associado com características sóciodemográficas distintas. Atos considerados fisicamente abusivos contra a criança são socialmente aceitos como práticas educativas na sociedade brasileira. Cardia 45, avaliando atitudes, normas culturais e valores em relação à violência em dez capitais brasileiras no ano de 1999, encontrou dados divergentes entre as capitais e explica que estas diferenças se devem aos hábitos culturais distintos de cada região, e não à presença ou ausência de violência.

Algumas limitações do estudo atual devem ser mencionadas. Por se tratar de um estudo de base populacional, o delineamento transversal é o mais indicado para mensurar a prevalência de histórico de violência na infância. Entretanto, os resultados através do auto-relato de adultos sobre experiências de violência na infância podem ter levado a viés de memória. Assim a prevalência do histórico de violência na infância pode estar subestimada. Uma avaliação mais proximal poderia render resultados mais robustos.

Outra limitação é com relação à falta de maior diversidade dos tipos de abuso sofridos na infância. As experiências abusivas resultantes da infância, incluídas no estudo, foram apenas abuso físico e testemunho à violência parental, não incluindo outras formas de abuso que podem co-ocorrer em famílias domesticamente violentas, tais como abuso psicológico, sexual e ou negligência parental.

Com relação à exposição à violência parental não foi realizada a observação se a mesma foi de pai-a-mãe ou de mãe-a-pai, o que, segundo a literatura, pode ter um impacto diferenciado nos resultados adversos apresentados na idade adulta. Diante disso, pesquisas futuras poderiam utilizar outros instrumentos mais completos, tais como a Conflict Tactics Scales: Parent-Child Version (CTSPC) concebida por Straus 46 e traduzida para utilização na população brasileira por Reichenheim \& Moraes 47. A CTSPC é composta por 22 itens divididos em 3 escalas e proporciona uma mensuração da disciplina parental não violenta, agressão psicológica e agressão física, além disso, contém questões suplementares sobre métodos de disciplina, negligência e abuso sexual.

Apesar destas limitações, o estudo atual foi o único estudo realizado sobre a prevalência de violência física e exposição à violência parental na infância com uma amostra representativa da população brasileira a partir de 14 anos de idade, de ambos os gêneros e sem exclusão de qualquer parte do território nacional (exceto aqueles mencionados na metodologia). A investigação seguiu padrões de estudos probabilísticos internacionais, priorizando um estrito rigor metodológico que resultou em dados de alta confiabilidade, confirmados pela ausência de vieses de seleção, mensurações adequadas aos fins propostos, além do controle intensivo de qualidade em todas as fases da pesquisa.

Em suma, o presente estudo mostrou que a ocorrência de abuso físico e exposição à violência parental na infância, relatado por adultos, é uma situação comum durante a infância nos lares brasileiros. Mais da metade da amostra, nacionalmente representativa tinha experimentado algum tipo de abuso físico na infância. Estudos futuros devem buscar examinar os possíveis impactos negativos e esclarecer possíveis fatores que possam contribuir no impacto da violência sofrida, tais como idade específica em que ocorreu, tipo de abuso, freqüência e duração, fonte (perpetrador) e a relação existente entre vítima e abusador, bem como outros tipos de experi- 
ências abusivas inter-pessoais não familiares e extra-familiares (na comunidade) devem ser consideradas, pois estes achados podem ajudar na implementação de estratégias de prevenção e programas de intervenções direcionados a esta população.

\section{Resumo}

O estudo objetivou avaliar a prevalência de abuso físico e exposição à violência parental na infância, segundo características sócio-demográficas. Um procedimento de amostragem de múltiplos estágios foi usado para selecionar 3.007 indivíduos de 14 anos de idade ou mais, entre 2005/2006, incluindo sujeitos de todas as regiões do país, assim, os resultados são nacionalmente representativos. Foram acessadas experiências de abuso físico (bater, bater com alguma coisa, queimar/escaldar, ameaçar/usar faca ou arma) e exposição à violência parental (testemunhou ameaça elou agressão física parental) na infância. Realizaram-se as análises através do teste qui-quadrado de Pearson. A prevalência de história de abuso físico na infância foi de 44,1\%, sendo que 33,8\% relataram história de abuso físico moderado e 10,3\% abuso físico severo. A prevalência de exposição à violência parental foi de 26,1\%, sendo que 7,5\% testemunharam violência parental moderada e 18,6\% testemunharam violência parental severa na infância. Análises combinadas entre estes dois tipos de violência demonstraram significativas associações entre elas. Estes resultados podem ajudar na implementação de estratégias de intervenção alertando profissionais de saúde para alta prevalência deste tipo de violência na população.

Violência Doméstica; Maus-Tratos Infantis; Pais

\section{Colaboradores}

D. V. Zanoti-Jeronymo participou do delineamento do estudo, revisão bibliográfica, análise dos dados, discussão dos resultados e foi redatora principal do artigo. M. Zaleski, I. Pinsky, R. Caetano foram autores do projeto que deu origem ao presente artigo tendo supervisionado as etapas de levantamento dos dados, supervisão do trabalho de campo, elaboração do banco de dados e revisão final do artigo. N. B. Figlie participou do delineamento do estudo, análise dos dados, discussão dos resultados e da redação e revisão final do trabalho. R. Laranjeira foi autor e coordenador geral do projeto de pesquisa que originou o presente artigo, contribuiu com a constante supervisão de todas as etapas de execução do trabalho.

\section{Agradecimentos}

Este trabalho constitui parte de uma pesquisa maior denominada I Levantamento Nacional sobre os Padrões de Consumo de Álcool na População Brasileira, conduzido pela Universidade Federal de São Paulo (UNIFESP), Unidade de Estudo de Álcool e Outras Drogas (UNIAD) e Secretaria Nacional Antidrogas (SENAD). Os autores agradecem à Secretaria Nacional Antidrogas pelo financiamento da pesquisa na qual se insere este estudo (SENAD - Convênio no ${ }^{\circ}$ 017/2003), e à Coordenação de Aperfeiçoamento Pessoal de Nível Superior (CAPES) pela concessão de bolsa para o primeiro autor durante a realização do doutorado, na UNIFESP, cujo trabalho de pesquisa originou este artigo. 


\section{Referências}

1. Krug EG, Dahlberg LL, Mercy JA, Zwi AB, Lozano R. World report on violence and health. Geneva: World Health Organization; 2002.

2. McAlister A. La violencia juvenil en las Américas: estudios innovadores de investigación, diagnóstico y prevención. Washington DC: Organización Panamericana de la Salud; 1998.

3. Malinosky-Rummell R, Hansen DJ. Long-term consequences of childhood physical abuse. Psychol Bull 1993; 114:68-79.

4. Moore CG, Probst JC, Tompkins M, Cuffe S, Martin $\mathrm{AB}$. The prevalence of violent disagreements in US families: effects of residence, race/ethnicity, and parental stress. Pediatrics 2007; 119 Suppl 1:S68-76.

5. Hussey JM, Chang JJ, Kotch JB. Child maltreatment in the United States: prevalence, risk factors, and adolescent health consequences. Pediatrics 2006; 118:933-42.

6. Bogat GA, DeJonghe E, Levendosky AA, Davidson WS, von Eye A. Trauma symptoms among infants exposed to intimate partner violence. Child Abuse Negl 2006; 30:109-25.

7. Bordin IAS, Paula CS, Nascimento R, Duarte CS Severe physical punishment and mental health problems in an economically disadvantaged population of children and adolescents. Rev Bras Psiquiatr 2006; 28:290-6.

8. Kernic MA, Wolf ME, Holt VL, McKnight B, Huebner CE, Rivara FP. Behavior problems among children whose mothers are abused by an intimate partner. Child Abuse Negl 2003; 27:1231-46.

9. Delaney-Black V, Covington C, Ondersma SJ, Nordstrom-Klee B, Templin T, Ager J, et al. Violence exposure, trauma, and IQ and/or reading deficits among urban children. Arch Pediatr Adolesc Med 2002; 156:280-5.

10. Garbarino J. An ecological perspective on the effects of violence on children. J Community Psychol 2001; 29:361-78.

11. Widom CS. Posttraumatic stress disorder in abused and neglected children grown up. Am J Psychiatry 1999; 156:1223-9.

12. Pfefferbaum B. Posttraumatic stress disorder in children: a review of the past 10 years. J Am Acad Child Adolesc Psychiatry 1997; 36:1503-11.

13. Harpaz-Rotem I, Murphy RA, BerkowitzS, Marans S, Rosenheck RA. Clinical epidemiology of urban violence: responding to children exposed to violence in ten communities. J Interpers Violence 2007; 22:1479-90

14. Smith JP, Williams JG. From abusive households to dating violence. J Fam Violence 1992; 7:153-65.

15. Garcia-Moreno C, Jansen HA, Ellsberg M, Heise L, Watts $\mathrm{CH}$. Prevalence of intimate partner violence: findings from the WHO multi-country study on women's health and domestic violence. Lancet 2006; 368:1260-9.

16. Briere J, Elliott DM. Prevalence and psychological sequelae of self-reported childhood physical and sexual abuse in a general population sample of men and women. Child Abuse Negl 2002; 27: 1205-22.
17. Henning $\mathrm{K}$, Leitenberg $\mathrm{H}$, Coffey $\mathrm{P}$, Bennett $\mathrm{T}$, Jankowski MK. Long-term psychological adjustment to witnessing interparental physical conflict during childhood. Child Abuse Negl 1996; 21: 501-15.

18. Heyman RE, Slep AMS. Do child abuse and interparental violence lead to adulthood family violence? J Marriage Fam 2002; 64:864-70.

19. Merril LL, Hervig LK, Milner JS. Childhood parenting experiences, intimate partner conflict resolution, and adult risk for child physical abuse. Child Abuse Negl 1996; 20:1049-65.

20. Bushman BJ. Human aggression while under the influence of alcohol and other drugs: an integrative research review. Curr Dir Psychol Sci 1993; 2:148-52.

21. Collins JJ, Messerschmidt PM. Epidemiology of alcohol-related violence. Alcohol Health Res World 1993; 17:93-100.

22. Kalmuss D. The intergenerational transmission of marital aggression. J Marriage Fam 1984; 46:11-9.

23. Carroll J. The intergenerational transmission of family violence: the long term effects of aggressive behavior. Aggress Behav 1977; 3:289-99.

24. Widom CS. Does violence beget violence? A critical examination of the literature. Psychol Bull 1989; 106:3-28.

25. Penna LHG, Santos NC, Souza ER. A produção científica sobre violência doméstica na área da saúde pública. Rev Enferm UERJ 2004; 12:192-8.

26. Souza ER, Minayo MCS. Análise das tendências da produção acadêmica sobre violência e acidentes. Rio de Janeiro: Centro Latino-Americano de Estudos sobre Violência e Saúde, Escola Nacional de Saúde Pública, Fundação Oswaldo Cruz; 2001.

27. Straus MA. Corporal punishment and prevention of physical abuse. Child Abuse Negl 2000; 124:1109-14

28. Schraiber LB, D'Oliveira AFPL, França-Junior I, Diniz S, Portella AP, Ludermir AB, et al. Prevalência da violência contra a mulher por parceiro íntimo em regiões do Brasil. Rev Saúde Pública 2007; 41:797-807.

29. Zaleski M, Pinsky I, Laranjeira RR, RamisettyMikler S, Caetano R. Intimate partner violence and contribution of drinking and sociodemographics: The Brazilian National Alcohol Survey. J Interpers Violence; no prelo.

30. Krug EG, Sharma GK, Lozano R. The global burden of injuries. Am J Public Health 2000; 90:523-6.

31. Straus MA, Gelles RJ. Physical violence in American families: risk factors and adaptations to violence in 8145 families. New Brunswick: Transaction Publishers; 1995.

32. Laranjeira R, Pinsky I, Zaleski M, Caetano R. I levantamento nacional sobre os padrões de consumo de álcool na população brasileira. http://www. uniad.org.br/docs/ILevAlcool.PDF (acessado em 10/Mar/2008)

33. Instituto Brasileiro de Geografia e Estatística. Pesquisa Nacional por Amostra de Domicílios. Rio de Janeiro: Instituto Brasileiro de Geografia e Estatística; 2004. 
34. Caetano R. Non-response in alcohol and drug surveys: a research topic in need of further attention. Addiction 2001;96:1541-5.

35. Zaleski M, Pinsky I, Caetano R, Sanches M, Cidade P Laranjeira R. I Brazilian Alcohol Survey: field work challenges. Rev Bras Psiquiatr; submetido.

36. Caetano R, Ramisetty-Mikler S, Rodriguez L. The Hispanic Americans Baseline Alcohol Survey (HABLAS): rates and predictors of DUI across Hispanic national groups. Accid Anal Prev 2008; 40:733-41.

37. Straus MA. Measuring intrafamily conflict and violence: the conflict tactics (CT) scales. In: Straus MA, Gelles RJ, editors. Physical violence in American families: risk factors and adaptations to violence in 8,125 families. New Brunswick: Transaction Publishers; 1990. p. 29-47.

38. Straus MA, Hamby SL, Boney-McCoy S, Sugarman DB. The Revised Conflict Tactics Scales (CTS2), development and preliminary psychometric data. J Family Issues 1996; 17:283-316.

39. Straus M, Gelles R. Societal change and change in family violence from 1975 to 1985 as revealed by two national surveys. J Marriage Fam 1986; 48: 465-79.

40. Thompson MP, Kingree JB, Desai S. Gender differences in long-term health consequences of physical abuse of children: data from a nationally representative survey. Am J Public Health 2004; 94: 599-604.
41. Caetano R, Field CA, Nelson S. Association between childhood physical abuse, exposure to parental violence, and alcohol problems in adulthood. J Interpers Violence 2003; 18:240-57.

42. Wolfner GD, Gelles RJ. A profile of violence toward children: a national study. Child Abuse Negl 1993; 17:197-212.

43. Assis SG, Avanci JQ. Labirinto de espelhos: a formação da auto-estima na adolescência. Rio de Janeiro: Editora Fiocruz; 2004

44. Reichenheim ME, Moraes CL, Szklo A, Hasselmann MH, Souza ER, Lozana JA, et al. The magnitude of intimate partner violence in Brazil: portraits from 15 capital cities and the Federal District. Cad Saúde Pública 2006; 22:425-37.

45. Cardia N. Pesquisa sobre atitudes, normas culturais e valores em relação à violência em 10 capitais brasileiras. Brasília: Secretaria de Estado dos Direitos Humanos, Ministério da Justiça; 1999.

46. Straus MA, Hamby SH, Finkelhor D, Moore DW, Runyan D. Identification of child maltreatment with parent-child conflict tactics scales: development and psychometric data for a national sample of American parents. Child Abuse Negl 1998; 22:249-70.

47. Reichenheim ME, Moraes CL. Psychometric properties of the Portuguese version of the Conflict Tactics Scales: Parent-child Version (CTSPC) used to identify child abuse. Cad Saúde Pública 2006; 22:503-15.

Recebido em 09/Jul/2008

Versão final reapresentada em 29/Jun/2009

Aprovado em 09/Jul/2009 